\title{
PERCEPCIÓN DE LOS DOCENTES DE EDUCACIÓN SECUNDARIA SOBRE LA CALIDAD DE LA ENSEÑANZA EN LA PROVINCIA DE ALBACETE
}

\section{PERCEPTION OF SECONDARY EDUCATION TEACHERS ON TEACHING QUALITY IN ALBACETE REGION}

\author{
Manuel Ángel González Berruga \\ manuel.gonzalez@pucese.edu.ec \\ Pontificia Universidad Católica del Ecuador Sede Esmeraldas (Ecuador)
}

Recibido: 03/02/2021

Aceptado: 30/06/2021

\begin{abstract}
Resumen:
Los docentes ocupan un lugar clave para el desarrollo de una educación de calidad en los centros de Educación Secundaria. La perspectiva del riesgo escolar es una propuesta que permite reconocer los factores que se encuentran en la construcción del fracaso escolar en el transcurso del estudiante por el sistema educativo. Se presenta una investigación descriptiva-interpretativa desde el enfoque teórico de las Pedagogía Productivas para conocer los factores de riesgo escolar desde la perspectiva docente de 11 centros de Educación Secundaria Obligatoria de la provincia de Albacete. Se observa una valoración baja de la atención a la diversidad y la contextualización de los aprendizajes. Los docentes planifican la práctica pedagógica con relación a un alumno medio. Los docentes con menos experiencia tienen una percepción más favorable en algunos factores sobre la calidad de la enseñanza. Los docentes de centros de niveles socioeconómicos y culturales altos presentan un estilo de enseñanza integrado y colaborativo en contraposición a los centros de nivel medio y bajo.
\end{abstract}

Palabras clave: Educación Secundaria, percepción docente, Pedagogías Productivas, riesgo escolar, calidad de la enseñanza.

\footnotetext{
Abstract:

Teachers have an important role in the development of an education of quality in Secondary Education schools. School risk perspective is a proposal that allows to recognize factors in the construction of school failure in the pass of the student through the educational system. It is presented a descriptive-interpretative research from the theorical perspective of Productive Pedagogies to know the school risk factors from teacher's perspective of 11 Secondary Education schools of Albacete region. It is observed a low perspective in diversity attention and learning contextualization. Teachers plan the pedagogical practice in relation to the average
} 
student in ordinary or PMAR classrooms. Less experienced teachers have a favorable perception in some factor about quality teaching. Teachers from schools with a high sociocultural and economic level present an integral and collaborative teaching style as opposed to middle and low-level schools.

Keywords: Secondary Education, teacher perception, Productive Pedagogies, school risk, teaching quality.

\section{Introducción}

Conseguir una enseñanza de calidad es un deseo compartido por instituciones y particulares que se ve frustrado por dinámicas y procesos que tiene su origen en la propia estructura escolar y se ven reforzados por factores de carácter social, económico y personal. A pesar de los esfuerzos realizados, el fracaso escolar en la ESO persiste en el conjunto de España y en particular en Castilla La Mancha. Según los datos para el curso 2017-18, en el caso del abandono escolar temprano, personas de 18 a 24 años que no terminan la secundaria y no se siguen formando, España es de los países de la Unión Europea que ha observado un mayor descenso de 2013 a 2018 con 5.7 puntos porcentuales, solo superado por Portugal con 7.1 puntos porcentuales, aun emerge un $17.9 \%$ de abandono, $14 \%$ para mujeres y $21.7 \%$ para hombres, 13.9 puntos menos que hace 10 años, con un 31.7\% en 2007-08, siguiendo a la cola de la Unión Europea cuya media se sitúa en $10.6 \%$. En el caso de Castilla La Mancha, la comunidad presenta un $20.5 \%$ en 2017 18 habiendo descendido 17.6 puntos porcentuales desde el curso $2007-08$ con un $38.1 \%$ de abandono (Ministerio de Educación y Formación Profesional, 2009 y 2019). Las cifras advierten de la dificultad que muchas personas tendrán para conseguir un trabajo o integrarse en otras esferas de la sociedad por la falta de capacidades, conocimientos y destrezas que se suponen imprescindibles.

El fracaso escolar presenta determinadas aristas y recovecos que se ocultan o salen a la luz en la dialéctica que se origina entre el intento de definirlo y la realidad particular, diversa y compleja que se nos presenta cuando se observa de cerca la historia escolar de cada alumno. Del aporte de Fernández Enguita (2011) se infiere que el intento de definir el fracaso escolar no puede recoger la profundidad y complejidad de un fenómeno y arroja la compleja cuestión de que si la escuela estuviera destinada a cumplir su cometido principal que es educar para que los sujetos puedan integrarse en la sociedad, no estaríamos hablando del fracaso escolar. Sin duda, el éxito o fracaso de los estudiantes depende de factores políticos, sociales, económicos, escolares, familiares y personales que favorecen y sancionan, ya sea por omisión o acción, voluntaria o involuntaria, de las personas involucradas, qué tipo de educación es recibida por cada persona. De las diferentes propuestas para acercarnos al fracaso escolar nos encontramos la perspectiva del riesgo escolar (Escudero, 2013) que nos permite comprender el fenómeno del fracaso como un continuo, desde una perspectiva temporal, y multifactorial, atendiendo a los diferentes elementos que generan situaciones singulares de riesgo escolar en las que se enmarca cada alumno, y multicausal, que nos permite acceder a las corresponsabilidades entre los actores con más o menos implicación y analizar los diferentes niveles de influencia en la construcción del fracaso escolar. Desde esta perspectiva, el docente cobra una especial relevancia por el hecho de situarse en un espacio de relación y de toma de decisiones que influye en múltiples elementos que explican, en parte, el fracaso escolar.

González González (2015) señala una serie de factores que guardan relación con la capacidad de actuación de los actores en los diferentes espacios que podemos identificar en una comunidad educativa como son el clima escolar, el currículum, la organización del centro y las relaciones con la comunidad. Los docentes juegan un rol fundamental en el engranaje y funcionamiento de 
estos elementos dentro del centro escolar puesto que intervienen de una manera $\mathrm{u}$ otra, $\mathrm{y}$ con mayor o menor intensidad, generando dinámicas que favorezcan o disminuyan las situaciones de riesgo escolar. Las relaciones entre estudiantes y docentes generan vínculos vitales y pedagógicos donde la participación en el aprendizaje (González González, 2010) o los vínculos emocionales son elementos clave del vínculo del alumnado con el centro (Ibrahim y El Zataari, 2020; Sointu, Savolainen, Lappalainen y Lambert, 2017) o las relaciones que los docentes mantienen con otros docentes y con el equipo directivo que permita el fomento de nuevas prácticas y proyectos o mejore la comunicación sobre los problemas educativos en el aula para mejorar los procesos educativos y sociales entre estudiantes y entre estos y los docentes (Ware y Kitsantas, 2010). Por otro lado, y como hemos señalado antes, el docente tiene la capacidad de adaptar el currículo prescriptivo a la realidad del centro y de la comunidad y de las necesidades y singularidades del alumnado, establecer formas de organización de tiempos y espacios para la mejora de la colaboración y la comunicación entre docentes y el equipo directivo y al fomento de la participación y colaboración con la comunidad educativa, desde las familias hasta otros actores que puedan participar de los procesos educativos.

En este sentido, es necesario conocer las ideas, creencias, valores, expectativas y motivaciones de los docentes para conocer de qué manera comprenden, perciben, sienten y valoran las experiencias educativas. El estudio de la percepción de los docentes desde diferentes enfoques y ámbitos es un campo prolífico (Álvarez, Álvarez, Castro, Campo y Fueyo, 2008; Chiner y Cardona, 2013; Colmenero, 2006; Meng, Muñoz y Wu, 2016; Oneke y Swain, 2014) que muestran, entre otras cosas, los elementos en los que tenemos que centrar nuestra atención, analizar, reflexionar e intentar modificar o transformar para alcanzar una educación de calidad.

Una de las propuestas para medir la calidad de la enseñanza es la de Hayes, Mills, Christie y Lingard (2006), sobre Pedagogías Productivas que permiten observar de manera integral los elementos que definen procesos educativos de calidad desde una perspectiva comunitaria, inclusiva (Allan, 2003), crítica, de justicia social y de progreso social (Lingard, Hayes y Mills, 2003; Lingard y Keddie, 2013) y que se ha utilizado en otras investigaciones (Bature y Atweh, 2020, 2016; Forbes et al., 2019; González Berruga y Escudero, 2018; Espinosa et al., 2018; Mills and Keddie, 2007; Mills y Goos, 2011; Mills et al., 2009, Marsh, 2007; Munns, 2007).

\section{Método}

La presente investigación tiene como objetivo conocer la percepción del profesorado sobre determinadas variables de los procesos de enseñanza-aprendizaje que pudieran identificarse como indicadores de riesgo escolar y explorar posibles diferencias atendiendo a los años de experiencia, a la enseñanza en grupos ordinarios o en grupos de atención a alumnado con dificultades, así como el nivel socioeconómico y cultural del entorno donde se inserta el centro escolar. Para ello, se toma una perspectiva descriptiva - interpretativa. Descriptiva en cuanto se utiliza un cuestionario para observar de manera epidérmica sus percepciones menos favorables sobre el proceso de enseñanza-aprendizaje e interpretativa puesto que se intenta ahondar a través de un focus group en aquellas cuestiones observadas como más urgentes después de analizar los cuestionarios.

Se desarrolla un cuestionario con $\mathrm{N}=26$ ítems con escala Likert con la opción de 1-Nada de acuerdo, 2 - Poco de acuerdo, 3 - No lo tengo claro, 4 - Bastante de acuerdo, 5 - Muy de acuerdo, atendiendo al marco teórico de las Pedagogías Productivas que se agrupan en las siguientes categorías: "Clima del Aula", "Calidad Intelectual de los Contenidos", "Reconocimiento y respuesta a la Diversidad" y "Contextualización de los aprendizajes". El cuestionario fue validado por expertos académicos en la materia y por docentes de etapa para conocer la adecuación y comprensión de los ítems. En la tabla 1 se pueden observar los items 
del cuestionario resumidos en cada dimensión. Se midió la percepción de la intencionalidad, esto es, el propósito y voluntad de los docentes por alcanzar el criterio estipulado y la percepción del logro, es decir, del grado de cumplimiento del criterio en su práctica pedagógica. El Alpha de Cronbach arroja una fiabilidad aceptable de $\alpha=.895$ para los resultados de intencionalidad (I) y el logro (L) de todos los ítems. Para cada categoría, "Calidad intelectual de los contenidos", $\mathrm{I}=.827, \mathrm{~L}=.834$; "Reconocimiento y respuesta a la diversidad", I=.650, L=.679; "Clima del aula", $\mathrm{I}=.669$, L=.692; "Contextualización de los aprendizajes", I=.274, L=.407.

Tabla 1.

Ítems del cuestionario y sus correspondientes categorías. Fuente: Elaboración propia.

\section{Calidad intelectual de los contenidos}

A.18. Contenidos interesantes y motivadores

A.1. Se trabajan contenidos fuera del libro

A.8. Comprensión profunda de contenidos

A.5. Trabajo grupal mejora la colaboración

A.3. Calidad por encima de cantidad de contenidos

A.13. Trabajo basado en proyectos

A.14. Uso del razonamiento y la reflexión

A.20. Relación contenidos entre asignaturas

A.12. Relación contenidos de la asignatura

A.16. Se conocen aprendizajes previos

A.22. Uso diferentes recursos

A.7. Explicación de contenidos y participación del alumnado

A.26. Uso de TICS para desarrollar habilidades complejas

A.24. Se estimula el diálogo y la discusión

\section{Reconocimiento y respuesta a la diversidad}

A.2. Se considera las diferentes capacidades e intereses

A.17. Recursos adaptados al ritmo del alumnado

A.15. Profesorado ayuda a quien lo necesita

A.6. Atención a todos los alumnos

A.23. Prestar atención a los más rezagados

A.9. Reconocer diferencias y asumir derechos

A.19. Buena relación alumnos profesor
A.4. Respeto diferencias personales
A.10. Normas de comportamiento aceptadas por todos
A.25. Atender aspecto cognitivo y emocional

Contextualización de los aprendizajes

A.11. Relación de contenidos con vida de los alumnos

A.21. Trabajo cooperativo para el sentido cívico

La muestra la compuso los docentes de 11 centros de Educación Secundaria de la provincia de Albacete. Se dividieron los centros según el nivel sociocultural y económico (SE) atendiendo a las características de las familias de los estudiantes que asisten al centro y a la oferta del centro. Estos datos se obtuvieron con ayuda de Inspección Educativa y el equipo directivo de los centros. Los centros de nivel alto tienen una población de nivel SE medio-alto y ofertan estudios de Bachiller con una fuerte voluntad de que los estudiantes promocionen a la Universidad; los de nivel medio tienen una población de nivel SE medio y la oferta va desde el Bachillerato a cursos de Formación Profesional Básica, Grado Medio y Superior, y los de nivel bajo presentan una 
población en su mayoría procedente de zonas rurales y con una orientación fuertemente dirigida a la Formación Profesional. En total participan $N=158$. Según los años de trabajo, que se dividen según el análisis de los textos de Bolívar (1999), Huberman (1996), Marquina, Yuni y Ferreiro (2017), los docentes con alta experiencia entre 21 y 36 años suponen el $45.5 \%$, seguidos por el rango de media experiencia entre 11 y 20 años con un $32.1 \%$ y de baja experiencia entre 0 y 10 años con un $22.4 \%$. Según el nivel socioeconómico se recogen un $24,1 \%$ de nivel alto, $22.8 \%$ de nivel medio y $53.2 \%$ de nivel bajo. Y con relación al grupo donde trabaja, un $78,5 \%$ en grupos ordinarios y un $21,5 \%$ en grupos del Programa de Mejora del Aprendizaje y del Rendimiento (PMAR).

El análisis de los datos cuantitativos se realizó a través del SPSS versión 23. Para observar los ítems referidos a los factores de la calidad de la enseñanza menos favorables se sumaron los porcentajes Muy de acuerdo y Bastante de acuerdo $(5+4)$ para observar a qué factores se les presta menos atención. Con estos datos, se restan los resultados de intencionalidad y logro para observar la diferencia entre la voluntad de alcanzarlo y la efectividad en el logro. La prueba Kolmogorov-Smirnov con corrección de Lilliefors arroja que los datos no se distribuyen de manera normal, aunque si se observa cierta tendencia normal en los histogramas por lo que se toma la decisión de analizar las diferencias significativas según variables recogidas en el cuestionario mediante el estadístico ANOVA y el Eta, que permite obtener el Eta-cuadrada (?2) para estimar la cuantía de esa diferencia (tamaño del efecto). Los estadísticos paramétricos, como ANOVA, son robustos con datos no normales, incluso más potentes que los no paramétricos para evitar errores de tipo I (Finch 2005; Lemeshko y Lemeshko, (2008). Para analizar la dimensionalidad de algunas escalas se utilizó el Análisis Factorial Exploratorio con rotación Varimax.

Una vez realizado el análisis de los datos recogidos por el cuestionario y observado los factores con una percepción más baja y, por lo tanto, como factores a tener en cuenta por ser parte de una situación de riesgo escolar para los estudiantes, se elaboró un focus group con docentes del centro de nivel SE medio para profundizar en la comprensión del fenómeno. El análisis se realizó a través de la codificación abierta para encontrar el sentido de cada unidad de significación por párrafos o frases (Schettini y Cortazzo, 2015) con la ayuda de programa Atlas.ti versión 6.

\section{Resultados}

Las frecuencias observadas en la figura 1 muestran la intención de los docentes, lo que se pretende enseñar al alumnado. En esta figura y en las que siguen, los ítems en azul corresponden a la dimensión "Clima del Aula", en amarillo a la dimensión "Calidad Intelectual de los Contenidos", en verde a la dimensión "Reconocimiento y respuesta a la diversidad" y en marrón a la dimensión "Contextualización de los aprendizajes". Por los resultados obtenidos, se identifican los ítems por debajo de $70 \%$ dentro de los factores de riesgo. Estos se refieren a tener en cuenta el nivel socioeconómico y cultural de los estudiantes para planificar las clases, a utilizar el trabajo cooperativo para promover el sentido cívico y la solidaridad entre estudiantes y al uso del trabajo por proyectos para el desarrollo de habilidades complejas y una actitud indagadora. Por dimensiones, se observa una mayor intencionalidad de los ítems referidos al "Clima del aula" y "Calidad intelectual de los contenidos" mientras que existe una baja intencionalidad en la "Contextualización de los aprendizajes" y "Reconocimiento y respuesta a la diversidad". 


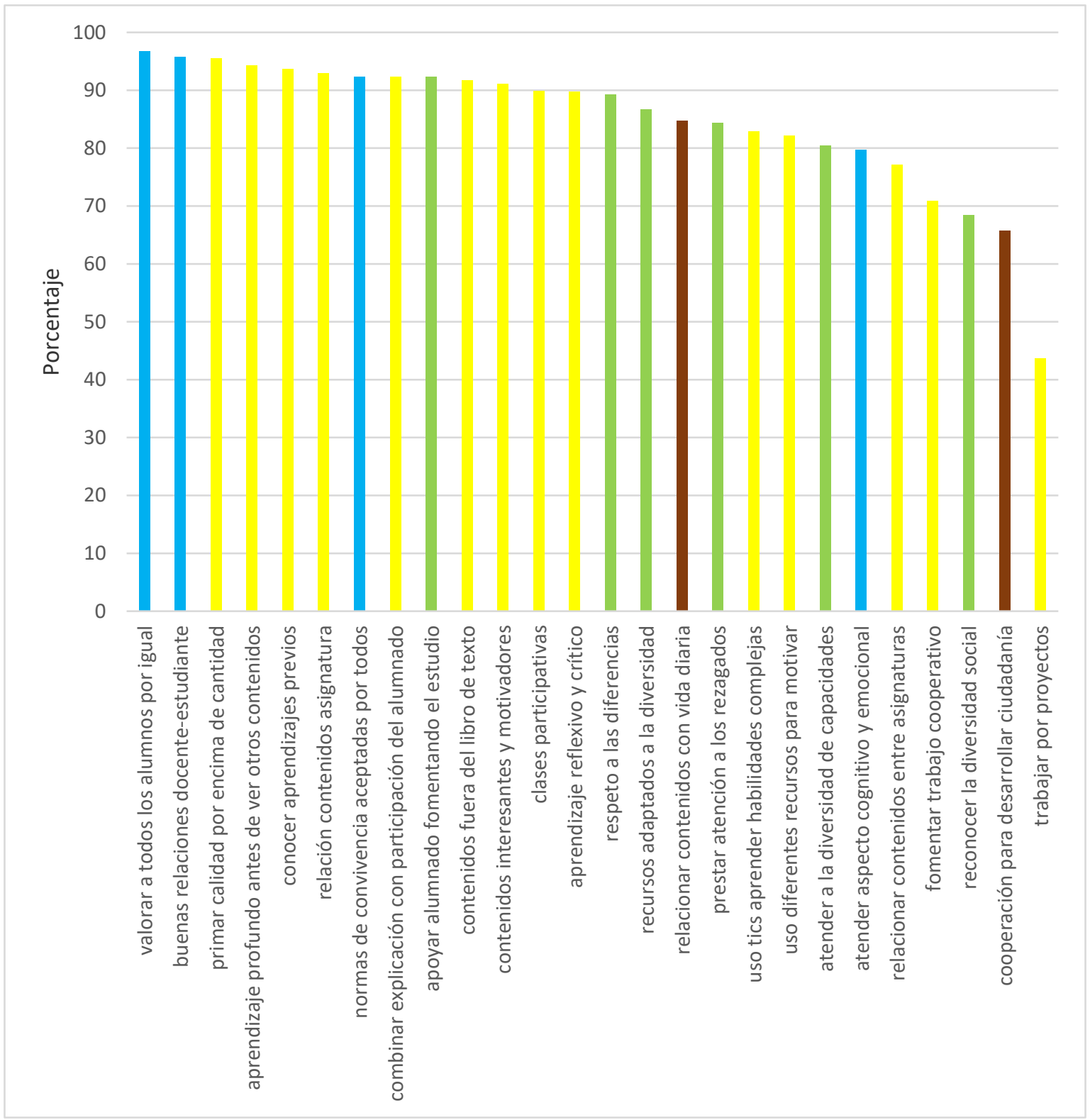

Figura 1. Intencionalidad docente en la planificación de la enseñanza. Fuente: Elaboración propia.

En cuanto al logro, lo que los docentes creen que alcanzan en la práctica pedagógica, en la figura 2 se observa que los porcentajes varían y los factores considerados de riesgo se ubican por debajo del $50 \%$. Estos corresponden a al desarrollo de una enseñanza que promueva el razonamiento y la reflexión sobre problemas, establecer relaciones entre los contenidos y actividades del aula y lo que ocurre en la vida cotidiana de los estudiantes, tener en cuenta la diversidad socioeconómica y cultural de los estudiantes al planificar las clases, facilitar el trabajo cooperativo y el apoyo mutuo, prestar atención a los que se quedan rezagados, al uso del trabajo cooperativo para promover el sentido cívico, atender a la diversidad de capacidades y motivaciones del alumnado y al uso del trabajo por proyectos para el desarrollo de habilidades complejas y una actitud indagadora. Por dimensiones, se observan los mismos datos que en la gráfica anterior, donde se observa una menor percepción de logro sobre la diversidad y la contextualización de los aprendizajes. 


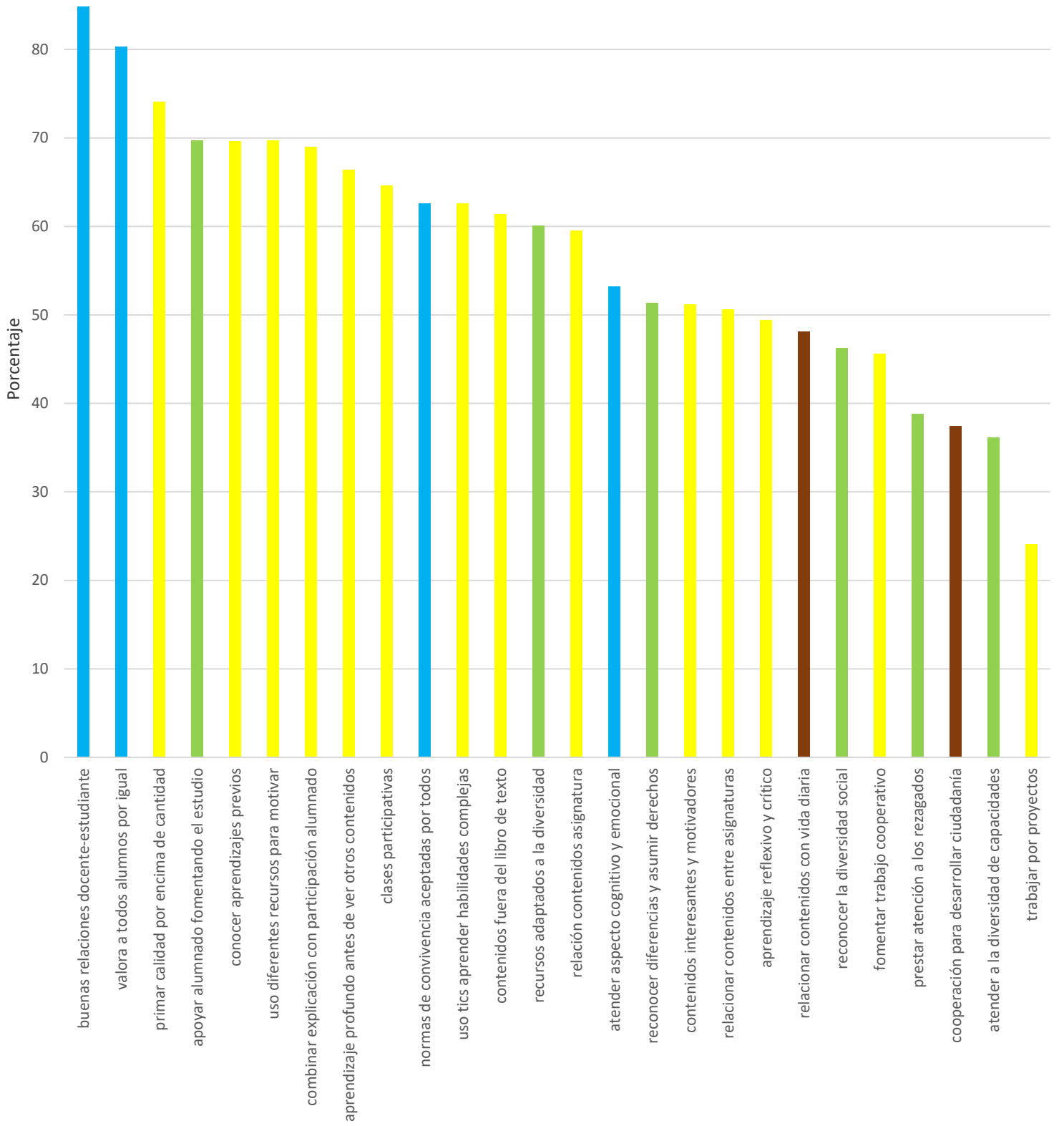

Figura 2. Logro docente en la práctica pedagógica. Fuente: Elaboración propia.

Al comparar la intencionalidad con el logro, se observa que la primera supera a la segunda, no hay ningún logro mayor que la intención de alcanzarlo, no se produce ningún resultado inesperado fuera de la planificación. Los factores donde se observa una brecha entre la intención y el logro con una mayor diferencia porcentual, por encima de 35 puntos porcentuales, se refieren a la atención de la diversidad de capacidades y motivaciones del alumnado, a prestar atención a los más rezagados para que no se quedan atrás, a desarrollar una enseñanza que promueva el razonamiento y la reflexión, a desarrollar contenidos y actividades que hagan el aprendizaje motivador e interesante, al reconocimiento y valoración de las diferencias en el aula, a la relación de las actividades y contenidos de clase con la vida cotidiana de los estudiantes y a facilitar el trabajo cooperativo (Figura 3). 


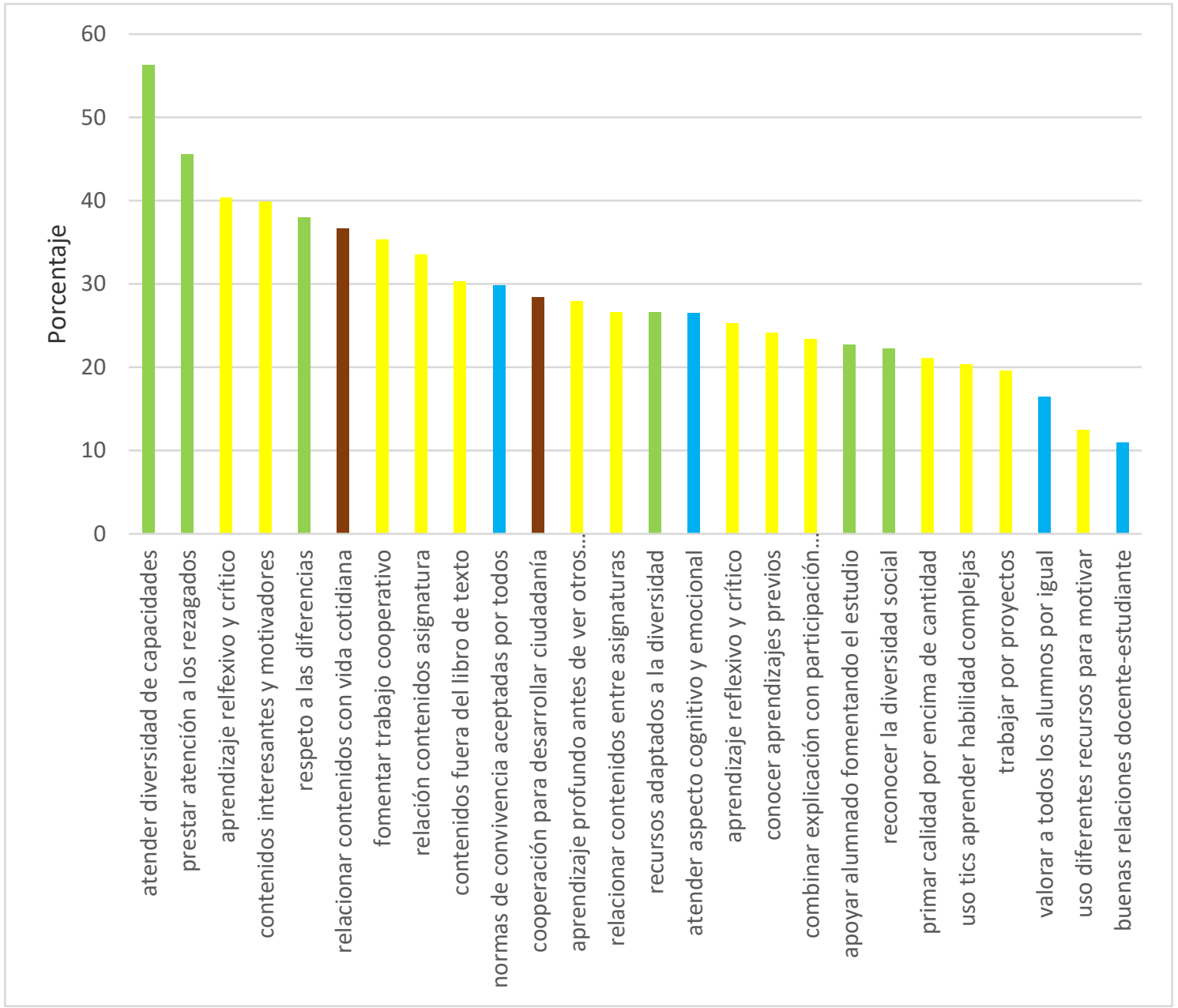

Figura 3. Diferencia entre intención y logro docente. Fuente: Elaboración propia.

A continuación, se estudian diferencias significativas según los años de experiencia, el trabajo en grupos ordinarios o en PMAR y según el SE. Según los años de experiencia, entre los docentes con experiencia baja y medio no se han encontrado diferencias significativas, por lo que la percepción de la enseñanza entre este grupo es similar no produciendo cambios significativos en el tiempo en cuanto a la percepción de la calidad de la enseñanza. Entre los docentes con experiencia media y alta se observan diferencias significativas que señalan un trabajo en grupo, con apoyo del docente, un buen clima en el aula, estimulándose el diálogo y la discusión trabajando actividades fuera del libro, y entre docentes de experiencia baja y alta, donde los primeros trabajan contenidos fuera del libro, estimulan la discusión y prestan más atención a los rezagados. Se observa como los docentes de experiencia media presentan un logro en su práctica educativa relacionada con factores protectores para una enseñanza de calidad (Tabla 2).

Tabla 2.

Diferencias significativas experiencia media-alta y baja-alta. Fuente: Elaboración propia

\begin{tabular}{|c|c|c|c|c|}
\hline & \multicolumn{2}{|c|}{ M } & \multirow[t]{2}{*}{ Sig. } & \multirow[t]{2}{*}{$\eta 2$} \\
\hline & Exp. Media & Exp. Alta & & \\
\hline Se trabajan contenidos fuera del libro & 3.88 & 3.48 & .005 & .064 \\
\hline Trabajo grupal mejora la colaboración & 3.60 & 3.17 & .006 & .061 \\
\hline Profesorado ayuda a quien lo necesita & 4.04 & 3.66 & .006 & .062 \\
\hline Buena relación alumnos profesor & 4.22 & 3.99 & .043 & .034 \\
\hline Se estimula el diálogo y la discusión & 3.92 & 3.49 & .005 & .063 \\
\hline
\end{tabular}




\begin{tabular}{|c|c|c|c|c|}
\hline & Exp. Baja & Exp. Alta & & \\
\hline Se trabajan contenidos fuera del libro & 3.83 & 3.48 & .034 & .042 \\
\hline Prestar atención a los más rezagados & 3.54 & 3.17 & .037 & .041 \\
\hline Se estimula el diálogo y la discusión & 4.03 & 3.49 & .003 & .083 \\
\hline
\end{tabular}

Atendiendo a lo que dan clase en grupos ordinarios y en PMAR se observa una mejor relación con los estudiantes de los primeros $(p=.042$, $22=.026)$ pero, en general, no se observan diferencias que caractericen un trabajo singular por impartir clase en cada grupo.

Entre los centros de nivel bajo y medio no se observa una caracterización diferenciada entre docentes de ambos centros en los dos factores que se observan diferencias significativas sobre trabajar contenidos fuera del libro $(p=.006$, ? $2=.062)$ y trabajar de manera grupal $(p=.026$, ?2=.041). En cambio, se observa una fuerte caracterización de los docentes de nivel alto con relación al nivel bajo y medio (Tabla 3). Los docentes del centro de nivel alto se caracterizan frente a los de nivel medio por respetar las diferencias personales, desarrollar normas de comportamiento, trabajar por proyectos, trabajar mediante el razonamiento y la reflexión, con contenidos interesantes, adaptarse al alumnado, trabajar de manera cooperativa con sentido cívico y atender al aspecto cognitivo y emocional; y frente a los de nivel bajo se caracterizan por reconocer y asumir los derechos de los estudiantes, desarrollar normas de comportamiento aceptadas por todos, relación contenidos de la propia asignatura y trabajar por proyecto, usar el razonamiento y la reflexión y trabajar de manera cooperativa con sentido cívico. Se observa como el docente percibe un mayor logro en factores determinantes para una educación de calidad, lo que se convierte en factores de riesgo en los centros de nivel medio y bajo, donde no se observa una caracterización diferente entre docentes.

Tabla 3.

Diferencias significativas nivel sociocultural y económico alto-medio y alto-bajo. Fuente: Elaboración propia.

\begin{tabular}{|c|c|c|c|c|}
\hline & \multicolumn{2}{|c|}{ M } & \multirow[t]{2}{*}{ Sig. } & \multirow[t]{2}{*}{$\eta 2$} \\
\hline & $\begin{array}{l}\text { Nivel } \\
\text { Alto }\end{array}$ & $\begin{array}{c}\text { Nivel } \\
\text { Medio }\end{array}$ & & \\
\hline Respeto diferencias personales & 3.87 & 3.44 & .016 & .078 \\
\hline Normas de comportamiento aceptadas por todos & 4.13 & 3.69 & .019 & .074 \\
\hline Trabajo por proyectos & 3.16 & 2.44 & .001 & .132 \\
\hline Uso de razonamiento y reflexión & 3.84 & 3.28 & .003 & .113 \\
\hline Recursos adaptados al ritmo del alumnado & 3.89 & 3.47 & .024 & .069 \\
\hline Contenidos interesantes & 3.74 & 3.33 & .036 & .060 \\
\hline Trabajo cooperativo para el sentido cívico & 3.58 & 3.11 & .027 & .066 \\
\hline \multirow[t]{2}{*}{ Atender aspecto cognitivo y emocional } & 3.76 & 3.42 & .048 & .053 \\
\hline & $\begin{array}{c}\text { Nivel } \\
\text { Alto }\end{array}$ & $\begin{array}{l}\text { Nivel } \\
\text { Bajo }\end{array}$ & & \\
\hline Reconocer diferencias y asumir derechos & 3.87 & 3.43 & .007 & .059 \\
\hline Normas de comportamiento aceptadas por todos & 4.13 & 3.62 & .002 & .081 \\
\hline Relación contenidos de la asignatura & 3.89 & 3.62 & .047 & .032 \\
\hline Trabajo por proyectos & 3.16 & 2.73 & .043 & .034 \\
\hline Uso de razonamiento y reflexión & 3.84 & 3.43 & .004 & .066 \\
\hline Trabajo cooperativo para el sentido cívico & 3.58 & 3.15 & .020 & .044 \\
\hline
\end{tabular}


El análisis factorial con rotación varimax permitió observar los factores que se anidan en torno a un componente. Las pruebas previas, test KMO (Kaiser-Mayer-Olikin) y el test de esfericidad de Bartlett, avalaron la aplicación del análisis. El test de Bartlett arroja un p valor de .000 lo que significa, al ser $p<0.05$, que las variables están suficientemente intercorrelacionadas. El valor del test KMO de 0.865 nos indica una alta interrelación que permite realizar el análisis. Emergen 6 componentes que agrupan los factores del cuestionario que explican el 59.329\% de la variabilidad (Tabla 4).

Tabla 4.

Análisis factorial exploratorio con rotación varimax. Fuente: Elaboración propia.

\begin{tabular}{cccc|ccc}
\hline \multicolumn{3}{c|}{ Autovalores iniciales } & \multicolumn{3}{c}{ Suma de rotación de cargas al cuadrado } \\
\hline & & $\%$ & $\%$ & & $\%$ \\
& Total & varianza & acumulado & Total & varianza & acumulado \\
\hline 1 & 8.066 & 31.025 & 31.025 & 2.672 & 10.276 & 10.276 \\
2 & 2.009 & 7.726 & 38.750 & 2.409 & 9.264 & 19.539 \\
3 & 1.537 & 5.912 & 44.662 & 2.153 & 8.280 & 27.819 \\
4 & 1.332 & 5.125 & 49.787 & 1.953 & 7.512 & 35.330 \\
5 & 1.304 & 5.014 & 54.801 & 1.780 & 6.846 & 42.176 \\
6 & 1.177 & 4.528 & 59.329 & 1.375 & 5.289 & 47.466 \\
7 & .956 & 3.676 & 63.005 & & & \\
8 & .878 & 3.377 & 66.382 & & & \\
9 & .837 & 3.220 & 69.602 & & & \\
10 & .783 & 3.013 & 72.615 & & & \\
\hline
\end{tabular}

En la tabla 5 se observa que la matriz de componentes no ha salido del todo limpia, ya que se considera que los ítems "Comprensión profunda de contenidos" y "Trabajo basado en proyectos" podrían ubicarse en otros componentes según los resultados arrojados. De igual manera, la matriz no arroja los mismos componentes del cuestionario quedando los ítems dispersos entre los diferentes componentes. Se observa un mayor peso en el componente 1 de la dimensión "Clima del aula siendo la dimensión" y en el componente 2 y 4 de la dimensión "Calidad intelectual de los contenidos".

Este peso de los ítems sobre el clima del aula en el componente 1 se corresponde con la valoración de esta dimensión por los docentes. La atención y el reconocimiento de la diversidad y se relaciona con el cumplimiento de las normas del aula y el establecimiento de buenas relaciones entre docentes y estudiantes. Los factores sobre la atención a la diversidad y la contextualización de los aprendizajes se dividen entre todos los componentes por lo que estos son factores asociados a otras variables y no guardan una importancia en sí misma. Un mayor peso al clima del aula va en detrimento de los factores sobre atención a la diversidad. Esto se relaciona con los datos observados donde la atención a la diversidad es el factor que menos se alcanza en el aula por los docentes. Se vislumbra la idea de una enseñanza en la que los estudiantes se tienen que adaptar a la clase para recibir una enseñanza de calidad. 
Tabla 5.

Ítems de logro docente agrupados en componentes principales. Fuente: Elaboración propia.

\begin{tabular}{|c|c|c|c|c|c|c|}
\hline \multirow[t]{2}{*}{ Ítems } & \multicolumn{6}{|c|}{ Componentes } \\
\hline & 1 & 2 & 3 & 4 & 5 & 6 \\
\hline Buena relación alumnos profesor & .667 & & & & & \\
\hline Valorar a todos los alumnos por igual & .664 & & & & & \\
\hline Normas de comportamiento aceptadas por todos & .656 & & & & & \\
\hline Reconocer diferencias y asumir derechos & .527 & & & & & \\
\hline Comprensión profunda de contenidos & .399 & & & & .381 & .246 \\
\hline Relación contenidos de la asignatura & & .654 & & & & \\
\hline Relación contenidos entre asignaturas & & .523 & & & & \\
\hline Aprendizaje reflexivo y crítico & & .520 & & & & \\
\hline Relación de contenidos con vida de los alumnos & & .441 & & & & \\
\hline Contenidos interesantes y motivadores & & .429 & & & & \\
\hline Trabajo basado en proyectos & & .393 & & .364 & .324 & \\
\hline Atender aspecto cognitivo y emocional & & & .844 & & & \\
\hline Prestar atención a los más rezagados & & & .496 & & & \\
\hline Cooperación para desarrollar ciudadanía & & & .462 & & & \\
\hline Profesorado ayuda a quien lo necesita & & & .439 & & & \\
\hline Clases participativas & & & .438 & & & \\
\hline Uso diferentes recursos para motivar & & & & .779 & & \\
\hline Uso de TICS para aprender habilidades complejas & & & & .700 & & \\
\hline Fomentar trabajo cooperativo & & & & .365 & & \\
\hline Conocer aprendizajes previos & & & & & .633 & \\
\hline Recursos adaptados a la diversidad & & & & & .516 & \\
\hline Calidad por encima de cantidad de contenidos & & & & & .388 & \\
\hline Se trabajan contenidos fuera del libro & & & & & & .556 \\
\hline Combinar explicación y participación & & & & & & .404 \\
\hline Tener en cuenta la diversidad social & & & & & & .349 \\
\hline Se atiende a la diversidad de capacidades & & & & & & .282 \\
\hline
\end{tabular}

Con el análisis de los datos cuantitativos se desarrolló un grupo focal para tratar las cuestiones más relevantes. Participaron cuatro docentes: de inglés (P1), matemáticas (P2), ciencias (P3) y lengua y literatura (P4). Se les comenzó preguntando sobre las diferencias entre la intencionalidad y el logro. Entendían que era normal estas diferencias.

Yo creo que hasta incluso es normal que no se ajuste. Porque entre lo que tú quieres hacer y lo que luego puedes hacer por " $x$ " circunstancias hay diferencia. Tratar de aplicar lo que quieres hacer, pero no siempre puedes llevarlo a cabo por múltiples razones. Por uno mismo y por otras cosas. (P.2)

Y las atribuyen a diferentes factores.

Hombre pues yo, puede haber circunstancias personales de uno mismo, de cómo se dan las clases, de cómo se adaptan las clases al grupo que tienes, que son grupos cambiantes, diferentes cada año, eh..., Imprevistos que puedan ir surgiendo, ... (P.2)

Siendo uno de los factores principales el grupo de estudiantes.

...por ejemplo, en primero de la ESO tienes grupos que son muy participativos digamos entonces que la, si la intención es llevar un ritmo y ajustarte a una programación eso se retrasa. Pero bueno eso no siempre no es tan importante siempre y cuando pueda servir de aliciente, de mantener esa curiosidad por la asignatura. En cambio, otros pueden ser grupos más pasivos y pueden determinarte. (P.2) 
El grupo es lo determinante. (P.1)

...antes de empezar todos creemos que los grupos van a ser ideales, ... (P.4)

Señalan la falta de condiciones para ejercer una práctica pedagógica adecuada y hacen alusión a la posibilidad de las condiciones ideales para la enseñanza con un grupo de estudiantes "perfecto", el bienestar propio, o el funcionamiento de los medios materiales.

Porque, claro, luego tú llegas y te imaginas un grupo perfecto en septiembre incluso aunque conozcas a los chavales y el ambiente y a ti mismo. Luego ni el grupo es perfecto ni tú estás siempre al cien por cien, ni los medios materiales de funcionan y claro eso pues va bajando, ... (P.1)

Todo cambia. Nada es lo mismo. Los chavales no son lo mismo en primero que en segundo, es que trabajamos con personas. Y ni nosotros somos los mismos, ni el centro, ni los materiales. (P.3)

Ellos crecen y cambian mucho. (P.4)

Lo que es más o menos fijo es la programación, pero tampoco es así. Las expectativas se tienen que ajustar a la normativa, pero luego dentro de eso e introduciendo las reformas que haya que introducir y con respecto a la experiencia del curso anterior luego ya poner en marcha eso... (P.2)

Y luego tienes que hacer ajustes con relación a todo eso sobre la marcha. (P.3)

Los docentes hacen alusión al grupo de estudiantes "perfecto" que interpretamos que se refieren a un grupo de estudiantes con características ubicados en la centralidad de lo que se consideraría un rendimiento y comportamiento adecuado e idóneo para el aprendizaje dentro del contexto educativo para la enseñanza conformado por la planificación docente y de los órganos de dirección. También señalan la adaptación de los docentes a los recursos materiales y la programación de la enseñanza a las necesidades que surgen, pero, teniendo en cuenta los datos anteriores, puede que los ajustes sigan realizándose con la idea del estudiante medio y no teniendo en cuenta la diversidad intrínseca del grupo de alumnos. En cuanto a la atención a la diversidad y la contextualización de los aprendizajes señalan la imposibilidad de alcanzar tal aspecto.

Atender a la diversidad es tarea imposible. Ahí sí que hay una intención, pero luego la realidad, los medios, el grupo que puede ser numeroso, atiendes a la diversidad media pero cuando tienen necesidades concretas es difícil porque tienes toda una clase a la que atender. (P.2)

Lo primero sería bajar la ratio. Si porque así al grupo lo puedes atender mejor y se puede prestar más atención a las características personales de cada alumno. Pero con ratios numerosas lo veo difícil y sobre todo si hay alumnos más concretos que necesitan un profesor de clases particulares, un profesor al lado para que no se disperse. (P.2)

Con más recursos se podría dar una mejor atención. (P.3)

Pero no sólo nosotros. Si un médico tiene menos pacientes y más recursos puedes atender mejor. Nosotros tenemos 30 personas y tenemos que atender a las 30 . Al final acabas dando aspirina a todos. Con menos alumnos podrías darle otras cosas, pero así es complicado. (P.1)

La imposibilidad de atender a la diversidad y personalizar la enseñanza la justifican por el elevado número de estudiantes en cada grupo y por la falta de recursos. En esta línea se observa como los docentes señalan la idea del alumno medio cuando se refieren a que se atiende a la diversidad media o cuando señalan que tienen que realizar la misma planificación didáctica para 
todos los estudiantes. Cuando surge un estudiante que no se adapta al grupo o que requiere una atención especial supone una complicación cuando la diferencia es una característica inherente de los estudiantes. Los recursos a los que se refieren son personales por encima de los materiales, si tuvieran menos estudiantes por aula podrían dar una atención más personalizada y atender a las necesidades de manera particular. Se observa una falta de autocrítica en el grupo de docentes que tienden a buscar la causa fuera de su práctica pedagógica y de sus posibilidades de actuación.

\section{Discusión y conclusiones}

Se observa la contextualización de los aprendizajes y la atención a la diversidad como factores de riesgo con dificultades para alcanzarse por parte de los docentes. Los docentes tienden a planificar con relación a un "alumno medio" por la falta de medios y docentes que permita bajar la ratio de estudiantes por grupo.

Según la percepción de la intención y el logro, los docentes valoran el clima del aula y la calidad de los contenidos sobre las otras dimensiones al igual que se observan en los trabajos de Grant, Stronge y Xu (2013), Koutrouba (2012), Meng, Muñoz y Wu (2016) y Sointu, Savolainen, Lappalainen y Lambert (2017) señalando la importancia de mantener un clima de clase y el establecimiento de relaciones cordiales y favorables entre estudiantes y profesores para conseguir una educación de calidad.

La atención a la diversidad presenta una valoración menor coincidiendo con los aportes de Álvarez, Álvarez, Castro, Campo y Fueyo (2008), Chiner y Cardona (2013), Colmenero (2006), Koutrouba (2012) y Oneke y Swain (2014), al igual que la contextualización de los aprendizajes relacionado con el aporte de Banet (2007).

De los datos emerge la idea de que el profesorado planifica la práctica educativa teniendo en mente el prototipo de un estudiante medio, propia de una práctica educativa basada en la transmisión de conocimientos (Hargreaves, 1996) para trabajar de la misma forma con todos cuando la realidad es que ningún estudiante es igual a otro por sus características personales, familiares o de desarrollo (Almeida y Albert, 2009). Los docentes entrevistados justifican la falta de atención a la diversidad en relación con aspectos externos a la acción docente (González-Gil, Martín-Pastor, Poy y Jenaro, 2016).

Los docentes con menos experiencia (baja y media) presentan una percepción más favorable sobre la calidad del aprendizaje, mostrando contenidos fuera del libro de texto y trabajando mediante el diálogo y la discusión, y sociales, mejorando las relaciones con los estudiantes, datos que se relacionan con los aportes de Chiner y Cardona (2013) y Pegalajar y Colmenero (2017) que observan que los profesores con menos experiencia tienen actitudes más favorables para la inclusión de los estudiantes. Esto puede deberse a que el profesorado con más experiencia continúa desarrollando modelos de enseñanza dirigidos y guiados por el libro de texto.

Los profesores que enseñan a grupos ordinarios y a PMAR presentan el mismo nivel de percepción sobre el proceso de enseñanza dato que se relaciona con el trabajo de Poon, $\mathrm{Ng}$, Wong y Kaur (2016), pero difiere con las aportaciones de por De Boer, Pijl y Minnaert (2011), Ernst y Rogers (2009) y Suk, Forlin y Mei (2007) que observan percepciones favorables en aquellos docentes que trabajan con estudiantes con necesidades de apoyo educativo. La idea de que el conjunto del profesorado planifica la enseñanza teniendo en mente las características de un estudiante medio se hace más visible ya que los docentes que se adscriben a cursos de PMAR presentan los mismos juicios y percepciones sobre el proceso de enseñanza. Si tenemos en cuenta el estilo de enseñanza observado, estos datos se relacionan con el aporte de BuliHolmberg y Jeyaprathaban (2016) quienes concluyen que los profesores con un estilo tradicional 
de enseñanza no tienen la capacidad para atender a los estudiantes con diferentes necesidades. Los datos obtenidos se explican por la falta de preparación de los profesores que atienden a estudiantes con diferentes necesidades (Leko y Brownell, 2009), esto es, el profesorado no dispone del conocimiento necesario para darse cuenta de que es posible y es necesario trabajar de manera diferente con estudiantes con diferentes necesidades.

El nivel sociocultural del centro se relaciona con la percepción de logro de los docentes emergiendo un estilo de enseñanza más integrado y colaborativo en los docentes del centro de nivel de nivel sociocultural y económico alto en detrimento al modelo de enseñanza de los institutos de nivel medio y bajo que presenta un modelo dirigido. Estos datos no se relacionan con la investigación de Razer, Mittelberg, Motola y Bar-Gosen (2015) que observaron que los profesores de institutos de secundaria de bajo nivel socioeconómico presentaban actitudes de atención a la diversidad más favorable que profesores de centro de nivel socioeconómico alto.

\section{Referencias bibliográficas.}

Allan, J. (2003). Productive Pedagogies and the challenge of inclusion. British Journal of Special Education, 30(4), 175-179.

Almeida, M. S. y Albert, J. R. (2009). Las concepciones de los profesores y la respuesta a la inclusión en Lisboa. Revista de Educación Inclusiva, 2(2). Recuperado de http://www.ujaen.es/revista/rei/linked/documentos/documentos/3-2.pdf

Álvarez, E., Álvarez, M., Castro, O., Campo, A. y Fueyo, E. (2008). Funcionamiento de la integración en la enseñanza secundaria obligatoria según la percepción del profesorado. Psicothema, 20(1), 56 - 62.

Banet, E. (2007). Finalidades de la educación científica en secundaria: opinión del profesorado sobre la situación actual. Enseñanza de las Ciencias, 25(1), 5-20.

Bature, I. J., y Atweh, B. (2020). Mathematics teachers reflection on the role of productive pedagogies in improving their classroom instruction. International Journal of Educational Methodology, 6(2), 319-335. https://doi.org/10.12973/ijem.6.2.319

Bature, I. J., y Atweh, B. (2016). Achieving Quality Mathematics Classroom Instruction through Productive Pedagogies. International Journal of Educational Methodology, 2(1), 1-18. Recuperado de https://files.eric.ed.gov/fulltext/EJ1167275.pdf

Bolívar, A. (1999). Ciclo de vida profesional de profesores y profesoras de secundaria: desarrollo e itinerarios de formación. En J. Cerdán y M. Grañeras (Ed.), Investigación sobre el profesorado (II) 1993-1997 (pp. 163-191). Madrid: Ministerio de Educación y Ciencia.

Buli-Holmberg, J. y Jeyaprathaban, S. (2016). Effective Practice in Inclusive and Special Needs Education. International Journal of Special Education, 31(1), 119 - 134.

Chiner, E. y Cardona, M. C. (2013). Inclusive education in Spain: how do skills, resources, and supports affect regular education teachers' perceptions of inclusion? International Journal of Inclusive Education, 17(5), 526 - 541. DOI: 10.1080/13603116.2012.689864

Colmenero, Ma J. (2006). Análisis de las percepciones del profesorado de Educación Secundaria sobre los procesos de atención a la diversidad: Su incidencia en la formación. Profesorado. Revista de Currículum y Formación del Profesorado, 10(2). Recuperado de https://www.ugr.es/ recfpro/rev102COL4.pdf

De Boer, A., Pijl, S., y Minnaert, A. (2011). Regular primary school teachers' attitudes towards inclusive education: A review of the literature. International Journal of Inclusive Education, 15, $331-353$. 
Ernst, C. y Rogers, M. (2009). Development of the inclusion attitude scale for high school teachers. Journal of Applied School Psychology, 25, 305- 322.

Escudero, J. M. (Coor.) (2013a). Estudiantes en riesgo, centros escolares de riesgo: Respuestas educativas al alumnado en situaciones de vulnerabilidad. Murcia: DM Editor.

Espinosa, A., Datukan, J. T., Butron, B. R. y Tameta, A. D. C. (2018). Perceptions of pre-service chemistry teachers on the utilization of productive lesson study as a framework for teaching and learning. International Journal for the Scholarship of Teaching and Learning, 12(1). https://files.eric.ed.gov/fulltext/EJ1172224.pdf

Fernández Enguita, M. (2011). Del desapego al desenganche y de éste al fracaso. Revista de la Asociación de Sociología de la Educación, 4(3), 255-269.

Finch, H. (2005). Comparison of the Performance of Nonparametric and Parametric MANOVA Test Statistics when Assumptions Are Violated. Methodology, 1(1), 27-38. DOI: 10.1027/1614-1881.1.1.27

Forbes, J. et al. (2019). Co/productive practitioner relations for children with SLCN: an affect inflected agentic frame. Discourse: Studies in the Cultural Politics of Education, 40(6), 859872. https://doi.org/10.1080/01596306.2018.1451303

González Berruga, M. A. y Escudero, J. M. (2018). Perspectivas de los Estudiantes Sobre el Aprendizaje en Educación Secundaria. Una Aproximación al Fracaso Escolar. Hallazgos21, 3(1), 34-56.

González González, Mạ T. (2015). Los centros escolares y su contribución a paliar el desenganche y abandono escolar. Profesorado. Revista de currículum y formación del profesorado, 19(3), 158-176. Recuperado de http://www.ugr.es/local/recfpro/rev193ART10.pdf

González González, Ma T. (2010). El alumno ante la escuela y su propio aprendizaje: algunas líneas de investigación en torno al concepto de implicación. Revista Iberoamericana sobre Calidad, Eficacia y Cambio en Educación, 8(4). Recuperado de https://revistas.uam.es/index.php/reice/article/view/4735/5169

González-Gil, F., Martín-Pastor, E., Poy, R. y Jenaro, C. (2016). Percepciones del profesorado sobre la inclusión: estudio preliminar. Revista Electrónica Interuniversitaria de Formación del Profesorado, 19(3), $11-24$.

Grant, L., Stronge, J. y Xu, X. (2013). A cross-cultural comparative study of teacher effectiveness: Analyses of award-winning teachers in the United States and China. Educational Assessment, Evaluation and Accountability, 25(3), 251 - 276.

Hargreaves, A. (1996). Cuatro edades del profesionalismo y del aprendizaje profesional. Seminario internacional sobre formación inicial y perfeccionamiento docente. Santiago de Chile.

Hayes, D., Mills, M., Christie, P. y Lingard, B. (2006). Teachers \& Schooling Making a Difference, Australia: Allen \& Unwin.

Huberman, M. (1996). Las fases de la profesión docente. Ensayo de descripción y previsión. Quaderns Digitals/Curriculum. Recuperado de http://www.quadernsdigitals.net/datos/hemeroteca/r_12/nr_186/a_2638/2638.html

Ibrahim, A. y El Zataari, W. (2020). The teacher-student relationship and adolescents' sense of school belonging. International Journal of Adolescence and Youth, 25(1.31), 382-395. 
Koutrouba, K. (2012). A profile of the effective teacher: Greek secondary education teachers' perceptions. European Journal of Teacher Education, 35(3). Recuperado de http://www.tandfonline.com/doi/full/10.1080/02619768.2011.654332?src=recsys

Leko, M. y Brownell, M. (2009). Crafting Quality Professional Development for Special Educators. What School Leaders Should Know. Teaching Exceptional Children, 42(1), 64 - 70.

Lemeshko, B. Y. y Lemeshko, S. B. (2008). Power and robustness of criteria used to verify the homogeneity of means. Measurement Techniques, 51(9), 950-959. http://dx.doi.org/10.1007/s11018-008-9157-3

Lingard, B., Hayes, D. y Mills, M. (2003). Teachers and Productive Pedagogies: contextualising, conceptualising, utilising. Pedagogy, Culture \& Society, 11(3), 399-424.

Marquina, M., Yuni, J., \& Ferreiro, M. (2017). Trayectorias académicas de grupos generacionales y contexto político en Argentina: Hacia una tipología. Archivos Analíticos de Políticas Educativas, 25(118).

Marsh, J. (2007). New literacies and old pedagogies: Recontextualizing rules and practices. International Journal of Inclusive Education, 11(3), 267-281.

Meng, L., Muñoz, M. A. y Wu, W. (2016). Teachers' perceptions of effective teaching: a theorybased exploratory study of teachers from China. Educational Psychology, 36(3), 461 - 480.

Ministerio de Educación y Formación Profesional (2009). Las cifras de la educación en España. Curso 2007-2008. Madrid: Secretaría General Técnica. Recuperado de https://www.educacionyfp.gob.es/servicios-al-ciudadano/estadisticas/indicadorespublicaciones-sintesis/cifras-educacion-espana/2007-08.html

Ministerio de Educación y Formación Profesional (2019). Datos y cifras. Curso Escolar 2019/2020. Madrid: Secretaría General Técnica. Recuperado de https://www.educacionyfp.gob.es/dam/jcr:b998eea2-76c0-4466-946e965698e9498d/datosycifras1920esp.pdf

Mills, M. y Keddie, A. (2007). Teaching boys and gender justice. International Journal of Inclusive Education, 11(3), 335-354.

Mills, M. y Keddie, A. (2013). Redistribution, recognition and representation: working against pedagogies of indifference. Pedagogy, Culture and Society, 21(3), 427-447

Mills, M. y Goos, M. (2011). Productive pedagogies in the mathematics classroom: Case studies of quality and equity. Mapping Equity and Quality in Mathematics Education. En Atweh, B., Graven, M., Secada, W. y Valero, P. (Eds.), Mapping Equity and Quality in Mathematics Education (pp. 479-491), EEUU: Springer.

Mills, M., Goos, M., Keddie, A., Honan, E., Pendergast, D., Gilbert, R., Nichols, K., Renshaw, P. y Wright, T. (2009). Productive Pedagogies: A Redefined Methodology for Analysing Quality Teacher Practice. The Australian Educational Researcher, 36(3) 67-87.

Munns, G. (2007). A sense of wonder: Pedagogies to engage students who live in poverty. International Journal of Inclusive Education, 11(3), 301-315.

Oneke, R. y Swain, K. (2014). Teachers' perceptions of students with special education needs in Cameroon secondary schools. International Journal os Special Education, 29(3), 101-110.

Pegalajar, M. C. y Colmenero, M. J. (2017). Actitudes y formación docente hacia la inclusión en Educación Secundaria Obligatoria. REDIE. Revista Electrónica de Investigación Educativa, 19(1), 84 - 97. 
Poon, K., Ng, Z., Wong, M. y Kaur, S. (2014). Factors associated with staff perceptions towards inclusive education in Singapore. Asia Pacific Journal of Education. DOI: 10.1080/02188791.2014.922047

Razer, M., Mittelberg, D., Motola, M. y Bar-Gosen, N. (2015). Israeli high school teachers' perceptions and attitudes towards a pedagogy of inclusion. International Journal of Inclusive Education, 19(9). Recuperado de http://dx.doi.org/10.1080/13603116.2015.1019373

Schettini, P. y Cortazzo, I. (2015). Análisis de datos cualitativos en la investigación social: procedimientos y herramientas para la interpretación de información cualitativa. Argentina: Universidad de La Plata.

Sointu, E., Savolainen, H., Lappalainen, K. y Lambert, M. (2017). Longitudinal associations of student-teacher relationships and behavioral and emotional strengths on academic achievement. Educational Psychology, 37(4), 457- 467.

Suk, C., Forlin, C., y Mei, A. (2007). The influence of an inclusive education course on attitude change of pre-service secondary teachers in Hong Kong. Asia-Pacific Journal of Teacher Education, 35, $161-179$.

Ware, H. y Kitsantas, A. (2010). Teacher and Collective Efficacy Beliefs as Predictors of Professional Commitment. The Journal of Educational Research, 100(5), 303-310, 